\section{The 3 Pillars of Health}

\author{
M. Castillo, Editor-in-Chief
}

$F_{b}$ or many years, I undervalued sleep. Why sleep when one can be up and about? Well, several things have recently caused me to change my mind. Our understanding of sleep and how it affects our brains and bodies is rapidly changing, and personal fitness devices help us keep track of these effects.

Approximately 2 months ago, I bought a personal fitness device; one of those plastic bands worn around a wrist that have lately been in the news. Most of these devices rely on accelerometers and altimeters (such as those used in the iPhone and iPad that allow one to play games by twisting and moving the devices) and store their data in small computers that then take this information to calculate different parameters. With regard to the types of physical activity analyzed, the performances of these devices vary and I tend to concentrate on trends rather than specific daily measures (but by now I know exactly how much I walk when on clinical service from my office to the reading room and how much I move during academic days).

With regard to sleep, my device does something called "sleep efficiency tracking." After communicating via Bluetooth and Wi-Fi with my telephone, I get to see the following: total sleep time, hours of deep and light sleep, and times and duration of periods in which I was awake. Every few days, it gives me messages informing that I am sleeping well or need to sleep more such as, "If you sleep more than 7 hours today, tomorrow you will feel more rested, confident, beautiful, and smart!" How can one resist such messages?

In addition (I have not tried it yet), the device has a smart waking alarm, meaning that it will wake me within 30 minutes of a selected time based on my REM cycles. Apparently, this eliminates the disconcerted feeling of being woken up in the middle of a dream. In reality, I think that most sleep information it provides is fairly vague but, again, trends are what count.

One message that my device has sent me several times states that sleep (the National Sleep Foundation recommends 7-9 hours per day for adults) helps one lose weight, and this does make sense. If one is not sleeping well, one is spending more time awake, which increases the chance of eating and induces fatigue that leads to more eating (especially carbohydrates) and lesser physical activity. Sleep deprivation produces a "double hit" to the brain in this regard: there is a sharp reduction in frontal lobe activity leading one to misjudge hunger and eat more junk and also leading to an exaggerated activation of reward centers (mostly the amygdalae) after eating. One meta-analysis of nearly 700 published studies showed that both adults and children who are short sleepers have an increased risk of obesity. ${ }^{1}$ In a different study, 12 men were allowed a full night of sleep ( 8 hours) followed by a partial night of sleep ( 4 hours); after the latter, the men were hungrier upon waking up and ate more during the day $(22 \%){ }^{2}$ Acute par-

http://dx.doi.org/10.3174/ajnr.A4025 tial sleep leads to increased serum levels of ghrelin (a hunger hormone) and decreased levels of leptin (a satiety hormone). Paradoxically, sleeping less leads to increased metabolism and extra burning of calories (but on average just a paltry 111 calories per day). However, the compensatory intake of food exceeds this amount and results in a net weight gain. ${ }^{3}$ Partial sleepers tend to consume approximately 600 more calories than full-night sleepers, with most of those calories coming from chocolate, desserts, and potato chips. ${ }^{4}$ Poor food choices are common in the sleep deprived. One of the authors of this last study speculated that adenosine, which is a brain metabolic by-product, possibly disrupts neural function by accumulating in the sleep-deprived brain. ${ }^{5}$ It is conceivable that adenosine accumulation affects the way we perceive food. Because sleep clears the brain of adenosine, sleep is the equivalent of chemically rebooting our brains.

Sleep must be terribly important because all animals on earth do it. A recent article published in Science starts to shed light on its importance. Rats undergoing natural sleep or sleep induced by anesthesia increased their brain's interstitial spaces by $60 \%{ }^{6}$ This leads to significant exchanges between CSF and interstitial fluid that result in increased rates of beta-amyloid clearance during sleep. Apparently, amyloid accumulates during the day and sleep disposes of it at night. This system is thought to be analogous to the lymphatic system that clears metabolic waste from the rest of the body, hence its name: glymphatic system (glial + lymphatic). Sleep promotes memory consolidation and that is why this process is abnormal in the elderly (who sleep less) and in those with diseases such as Alzheimer, in which amyloid accumulates.

The term "glymphatic system" derives from the fact that the glial cells are the ones mostly responsible for the exchange of fluids through their membranes. The glymphatic system is a highenergy system and that is why it cannot work when the brain's energy is being used for other activities such as being awake. Wakefulness decreases the brain's fluid exchange capacity by approximately 95\%. Norepinephrine probably serves to control this fluid exchange. The authors of the Science article speculated that increased norepinephrine when awake results in increased cell volume and conversely decreased interstitial volume. Animals given adrenergic receptor antagonists show increased CSF influx into their brain's interstitial space. Thus, rather than sleep, it is probably wakefulness that primarily regulates the glymphatic system. The problem is that once amyloid starts to accumulate in the brain, sleep cycles are further upset, and the insomnia that occurs with Alzheimer and other dementias further exacerbates the lack of clearance of the brain's noxious substances.

Although the concept of the glymphatic system is today believed to be related only to amyloid, it is possible that it also plays a role in the disposal of other proteins such as tau and alphasynuclein that are involved in other neurodegenerative disorders, especially Parkinson disease. Other possible implications of this mechanism are the increase in migraines and seizures in patients who sleep poorly. It is conceivable that, in the future, instead of treating these diseases directly, we could design medications that enhance the functioning of the glymphatic system and radiotracers that could be used in vivo to assess their effects. How CSF gets 
into the brain is still unclear, possibly through its external and ventricular surfaces and the perivascular spaces.

PET imaging with the Pittsburgh compound (PiB-PET) has shown that shorter sleep duration is associated with higher amyloid brain burden. ${ }^{7}$ Other studies have shown that unfragmented sleep reduces the risk of Alzheimer disease (and decreases the development of neurofibrillary tangles) and diminishes normal age-related cognitive decline. ${ }^{8}$ Apnea is another factor that prevents adequate sleep consolidation and though it was thought to affect mentation by vascular effects on the brain, the worse cognition associated with it could be caused by interruptions of the glymphatic system function. Of course, one's inability to consolidate sleep is multifactorial and includes comorbidities and genetic and environmental factors among others. Perhaps information gathered by personal fitness devices on the sleep patterns of millions of users will shed more light onto the relationship between sleep and successful aging. Over $60 \%$ of all adults report trouble sleeping at night, thus the implications of the relationship between sleep and cognition are staggering.

Regardless of any scientific evidence, sleep is a hot item and even a new, short e-novella by famous (and very good) author Karen Russell deals with the subject. ${ }^{9}$ In this short book, America experiences an epidemic of insomnia and a large corporation decides that sleep is a commodity. Healthy sleepers are urged to "donate" their sleep to those less fortunate. The fact that sleep is indeed a commodity is now being used by industries successfully. Personal fitness and activity tracking devices generated over US $\$ 290$ million last year in sales and this is expected to double soon. Today, there are so many brands that manufacture these personal fitness devices that choosing one is difficult, particularly if one did not sleep well the night before.

So, what did I learn about my sleep in the last 2 months of wearing my Up24 band? Well, only good news. I fall asleep faster and sleep longer than I thought and have longer periods of deep sleep than others in my "team" (yes, you cannot only compete for the most activities but also for the most and best sleep). I have also become more aware as to how much people care about how I well sleep: hotels offer me better mattresses, more pillow choices, highefficiency sheets and pillow covers, mood lightning and soothing sounds, calming pulse-point oils, and some will even call before I go to sleep to remind me to turn my electronic devices off (the blue wavelength light these devices emit affects the secretion of melatonin more powerfully than any other type of light). Airlines lagged behind hotels and now that, at least in business class, the food is improving, they are concentrating on sleep and offering natural-fiber bedding, flat-bed seats, noise-cancellation headphones, and "radio" stations with only white noise. Why the industry is doing all of this is not clear to me and in a recent article in the New York Times, the executive director of the Harvard Medical School Division of Sleep Medicine said, "Sleep is the enemy of capitalism, you can't produce or consume when you're asleep."10 In the same article, Dr. Sanna also says that we need to stop thinking of sleep as a commodity and a lifestyle choice, but rather as the third pillar of health together with diet and exercise. Could it be that living and sleeping better and longer is becoming more important than just accumulating stuff? I do not think so, but to- night I will go to sleep earlier hoping that it will help me be a smarter and healthier neuroradiologist tomorrow.

\section{REFERENCES}

1. Cappuccio FP, Taggart FM, Kandala NB, et al. Meta-analysis of short sleep duration and obesity in children and adults. Sleep 2008; 31:619-26

2. Brondel L, Romer MA, Nouques PM, et al. Acute partial sleep deprivation increases food intake in healthy men. Am J Clin Nutr 2010;91:1550-59

3. Markwald RR, Melanson EL, Smith MR, et al. Impact of insufficient sleep on total daily energy expenditure, food intake, and weight gain. Proc Natl Acad Sci U S A 2013;110:5695-700

4. Greer SM, Goldstein AN, Walker MP. The impact of sleep deprivation on food desire in the human brain. Nat Commun 2013;4:2259

5. O'Connor A. How sleep loss adds to weight gain. New York Times. August 6, 2013. http://well.blogs.nytimes.com/2013/08/06/how-sleeploss-adds-to-weight-gain. Accessed March 27, 2014

6. Xie L, Kang H, Xu Q, et al. Sleep drives metabolite clearance from the adult brain. Science 2013;342:373-77

7. Malkki M. Alzheimer disease: sleep alleviates AD-related neuropathological processes. Nat Rev Neurol 2013;9:657

8. Lim, AS, Yu L, Kowgier M, et al. Modification of the relationship of the apolipoprotein $\mathrm{E} \varepsilon 4$ allele to the risk of Alzheimer disease and neurofibrillary tangle density by sleep. JAMA Neurol 2013;70: $1544-51$

9. Russell K. Sleep Donation. New York: Atavist; 2014

10. Rosenbloom S. From airlines to hotels, a quest to help you sleep. New York Times. March 26, 2014. http://www.nytimes.com/2014/03/30/ travel/from-airlines-to-hotels-a-quest-to-help-you-sleep.html. Accessed March 27, 2014

\section{EDITORIAL}

\section{Point-TAR: A Useful Index to Follow-Up Coiled Intracranial Aneurysms?}

\author{
L. Pierot, J. Fiehler, and P. White
}

$T$ he Matrix and Platinum Science (MAPS) trial results were recently published showing that Matrix $^{2}$ coils were not inferior (and not superior) to bare metal coils (BMC) in the treatment of ruptured and unruptured intracranial aneurysms. ${ }^{1}$ In the trial, 626 patients were enrolled in 43 investigational sites from March 2007 to October 2009. Besides being designed to compare the results of aneurysm treatment with Matrix $^{2}$ and BMC, MAPS was also designed to evaluate a composite clinical outcome measure designated as "target aneurysm recurrence" (TAR) that was defined as occurring when a patient experienced any of the following conditions after his or her initial aneurysm coiling: 1) target aneurysm (re)hemorrhage, 2) target aneurysm retreatment, or 3) death from unknown cause.

As demonstrated by previous studies, there was no significant difference between Matrix ${ }^{2}$ and BMC regarding aneurysm occlusion (evaluated with modified 3-grade Raymond scale) by core lab evaluation at the end of the procedure and at 12 months. ${ }^{2,3}$ There was also no significant difference in the arms with respect to change in aneurysm occlusion evaluated with a 3 -grade scale (bet-

http://dx.doi.org/10.3174/ajnr.A4119 